\title{
RADIATION DAMAGE TO METALS AND ASSOCIATED PROBLEMS
}

$I^{\mathrm{N}}$ $\mathrm{N}$ the course of the annual autumn meeting of the Institute of Metals, held this year in Buxton, a discussion was held on September 22 on the subject of "Radiation Damage in Metals". The Metal Physics Committee of the Institute had arranged this dis. cussion under the chairmanship of Prof. R. W. K. Honeycombe, professor of physical metallurgy in the University of Sheffield, and speakers were drawn from the Motallurgy Division of the Atomic Energy Research Establishment.

Dr. M. J. Makin spoke about the effect of radiation on the mechanical properties of metals and the importance of knowledge of this phenomenon when undertaking the building of nuclear reactors, much of the structure of which is inevitably exposed to a heavy flux of neutrons. An experiment in which the mechanical properties of nickel were tested before and after irradiation showed, in respect of the irradiated sample, a considerable increase in yield stress, some reduction of ductility and a general lifting of the stress-strain curve. The rate of workhardening was less in irradiated material than in the original. The mechanical properties of irradiated specimens recorded at different testing temperatures showed that the results obtained were very dependent upon temperature, while a further variation in these properties was related to the temperature at which the irradiation took place. Some of the irradiation effects can be annealed out. Experiments with copper show that $300-400^{\circ} \mathrm{C}$. is a suitable range, corresponding to an activation energy of approximately $2 \mathrm{eV}$. With nickel, the range is $350-400^{\circ} \mathrm{C}$., suggesting that the activation energy is about the same as that of self-diffusion. The temperature indicated for nickel is rather lower than that at which cold work anneals out and, further, the reduction of ductility by irradiation is much less than that brought about by cold work.

The effect produced by irradiation on mechanical properties appears to resemble more closely the change produced by the addition of an alloying element than, as had been suggested originally, that induced by cold work. Irradiation of material with a ductile-brittle transformation raised the transition temperature. Work on single crystals of ductile metals shows that the rate of increase of yield stress is not linear; the effect appears to be proportional to the $\frac{1}{3}$ power of the neutron dose, and there is no evidence of a saturation point. Efforts have been made to picture a mechanism involving the displacement of atoms by fast neutrons. Thus, Brinkman, discussing copper, has suggested that the knocked-on atom actually melts a very small region of the crystal and that solidification is so rapid that the atom will not return to its original position. Another suggestion is that the copper atoms knocked out of the lattice migrate in clusters, forming a pseudoprecipitate which is attached to dislocations.

Discussing techniques used in studying radiation damage, Mr. O. Plail said that the main divisions of this study-irradiation and subsequent examination -both call for special experimental techniques. and scientific apparatus of novel types. It is necessary in the first place to avoid damage to the pile and, in the second, to carry out delicate tests by remote control on specimens which are strongly radioactive.
Mr. Plail's contribution clearly demonstrated the demands now made on technicians and apparatus constructors and how well these demands have been met.

Dr. P. C. L. Pfeil dealt particularly with the preparation and microscopic examination of irradiated specimens. The microscope used is of the usual inverted type, but the optical system following the objective is replaced by a high-definition television camera. The camera is connected to a large monitortube mounted in a conventional cabinet, and the image of the specimen is displayed on this monitor. A very high standard of resolution has been attained, photographs of the screen image (magnification, $\times 1,000$ ) being remarkably clear. Dr. Pfeil discussed the microstructures revealed by such examination and mentioned that a Russian delegate at the Geneva Conference reported that a sample of alloy containing alpha-uranium and gamma-uranium became 100 per cent gamma-phase when irradiated at a temperature at which it would otherwise have remained two-phase. There is a difference of opinion as to the mechanism involved in this change, but Dr. Pfeil favours the view that the presence of a body-centred-cubic nucleating agent will initiate the growth of a small region of body-centred-cubic structure among atoms which are cooling after the intense local heating in the track of a particle.

Dr. H. M. Finniston, head of the Metallurgy Division of the Atomic Energy Research Establishment, spoke about radiation damage in uranium and the marked dimensional changes observed in irradiated specimens. It has been found possible to study the dimensional changes by using a thermal analogy, and, although the phenomena are entirely different, the analogue method has given valuable information. It centres around the alpha-beta change which occurs at $650^{\circ} \mathrm{C}$. and consists of repeated thermal cycling between 600 and $650^{\circ} \mathrm{C}$. When this method was applied to rod specimens prepared in different ways, some specimens bent, and others grew lengthwise or suffered severe surface wrinkling. These effects can be explained by considering how the different fabrication processes or treatments have affected crystal orientation in the rods, since uranium shows pronounced erystal anisotropy. The closeness of the analogy is demonstrated by the effect of pile irradiation on single-crystal specimens of uranium which grow in the direction of the preferred axis. Dr. Finniston discussed the possible forms in which nuclear fuel might be prepared in future. He also referred to the difficulties of studying the properties of plutonium, which has six phase changes against the three in uranium.

Dr. J. H. O. Varley discussed radiation damage in jonic solids with particular reference to the alkali halides and methods of evaluating $\sigma$, the ionization cross-section. The investigating tool used is the colouring of the halide crystals on irradiation ${ }^{1,2}$. The colouring can be partly bleached out by light and more thoroughly by heat. The 'temporary' colouring (bleached by light) corresponds to displacements of electrons in the lattice, and the 'permanent' colouring (fast to light) is associated with displacement of atoms. The measured cross-section determined from the number of permanent colour centres per c.c. 
produced by a given bombardment is of the order $3 \times 10^{-21} \mathrm{~cm} .^{2}$. The cross-section calculated on the assumption that displacements are produced by direct collision with atomic nuclei lies between $5 \times 10^{-23}$ and $10^{-22} \mathrm{~cm} .{ }^{2}$. The measured cross-section gives a lower limit to the value pertaining to the principal displacement mechanism because there is not necessarily a one-one correspondence between the number of displacements and the number of colour centres produced. Dr. Varley used the relation between the cross-section $\sigma_{p}$ for a $p$-fold ionization of a given atom or ion and the cross-section $\sigma_{1}$ for a single ionization in the approximate formula

$$
\sigma_{p} \sim \sigma_{1}\left(\frac{\sigma_{1}}{A}\right)^{p-1}
$$

where $A$ is the 'area' of the atom. From the rangeenergy relation obtained experimentally, it appears that $\sigma_{1}$ is of the order $10^{-17} \mathrm{~cm}^{2} ; A$ can be taken as $\sim 10^{-15} \mathrm{~cm}^{2}$. By the substitution

$$
\sigma_{p} \sim 10^{-17}\left(10^{-2}\right)^{p-1}
$$

since $\sigma_{p} \sim 10^{-21} \mathrm{~cm}^{2}$, the approximation gives $p=3$ as the number of electrons stripped from one ion. It is thus indicated that the removal of three electrons from a given negative ion is a sufficiently probable process to account for the number of colour centres and, consequently, for displacements produced, if it be assumed that each one of these three. fold ionized ions is ejected from its lattice site and trapped at some other site in the crystal. The endstate can thus be halogen atoms in interstitial positions, trapped in positive-ion vacancies or at dislocations, and electrons trapped in the sites vacated by the halogen ions, these electrons giving rise to the colour centres. Dr. Varley concluded by remarking that vacancy pairs can lead to fast bleaching by acting as scavengers, but there is still much to be elucidated.

J. F. HINSLEY

${ }^{1}$ Varley, J. H. O., J. Nuclear Energy, 1, 130 (1954).

${ }^{2}$ Varley, J. H. O., Nature, 174, 886 (1954).

\section{THE NATIONAL GEOGRAPHIC SOCIETY_PALOMAR OBSERVATORY SKY ATLAS}

T HE first section of the Palomar Sky Atlas has recently been distributed to subscribers. This Atlas is being prepared from a survey of the sky north of declination $-27^{\circ}$, made with the 48 -inch Schmidt telesceis on Mount Palomar. This area of the sky has been photographed on 879 pairs of plates ; each plate of size 14 in. $\times 14$ in. covers an area of $7 \times 7$ degrees. The plate centres are separated by $6^{\circ}$, so that adjacent plates overlap by $\frac{1}{2}$ degree.

Each field is photographed in two colours in immediate succession. The blue exposures are obtained on the Eastman 103a0 emulsion, with a wave-length range of from $3500 \mathrm{~A}$. (the short-wave transmission limit of the correcting plate) to $5000 \mathrm{~A}$., the maximum sensitivity being at about $4100 \mathrm{~A}$. The red exposures are obtained on the Eastman $103 a E$ emulsion, combined with a red 'Plexiglass' filter, with a wave-length range of from $6200 \mathrm{~A}$. (the short-wave cut-off limit of the filter) to $6700 \mathrm{~A}$., the maximum sensitivity being at about $6500 \mathrm{~A}$.

The exposure times for each series were chosen to reach the faintest stars which can be recorded by the telescope under average observing conditions. These times were separately determined for each shipment of plates and range from 10 to $15 \mathrm{~min}$. for the blue exposures and from 40 to $60 \mathrm{~min}$. for the red. The photographic magnitude limit is $21 \cdot 1$ for the blue plates and 20.0 for the red. The magnitudes of stellar images on the blue plates are approximately on the same system as that of the international photographic magnitudes, and those of the images on the red plates are approximately on the same system as the red magnitudes of Kron and Smith. The differences between blue and red magnitudes of stars on the survey plates are approximately $\mathbf{1 . 6}$ times their international colour indices. Stars having an international colour index of 0.7 appear about equally bright on the blue and red plates. The scale of the plates is $67 \cdot 1$ seconds of are per millimetre.

Each pair of photographs of a field was inspected for quality before being accepted for reproduction in the atlas. If one member of a pair was rejected, the area was re-photographed in both colours. Many fields had to be photographed several times before both plates were passed as acceptable.

The photographs were first reproduced as positive transparencies. The photographic prints are in the form of negative reproductions, because they alone permit of satisfactory preservation of the plate limit. The prints were made by the Graphic Arts Facilities of the California Institute of Technology. Care has been taken to ensure that the faintest details at the limits of the plates are reproduced even at the price of inadequate reproduction of bright objects, so that the images of faint stars, galaxies and nebulosities, which are beyond the reach of smaller instruments, are preserved.

After extensive tests and trials, Eastman Kodak Finegrain Positive glass plates were selected for the positive transparencies and Eastman Kodak Unicontrast double-weight paper for the negative prints. The contrast of the paper is gamma $2 \cdot 0$, practically independent of development. The original plates show considerable variation in background density, which depends upon the sky brightness. Original plates with background densities less than $0 \cdot 3$ and greater than 0.9 were found to give inferior results and were rejected. The most satisfactory reproduction was found to be obtained by printing all positives to a density of $1 \cdot 4$. The paper prints were found to be most satisfactory with a density of 0.2 or slightly higher.

On the back of each print is stamped the nominal position of the plate centre for epoch 1855. In the north-east corner of each photograph is a label giving the serial number of the plate, the date of the exposure and the 1950 co-ordinates of the field centre.

The survey was made financially possible by generous grants from the National Geographic Society, which provided the photographic materials and equipment, the salaries of the personnel employed on the survey, and the cost of producing two contact positives on glass of each survey photograph. The 